\title{
Augmented plate causes bone resorption in reconstruction of large segmental femur defects with induced membrane technique
}

\section{Shengpeng Yu}

Army Medical University

Bao Yin

Dujiangyan Medical Ceter

\section{Shulin Wang}

Army Medical University

Chao Jia

Army Medical University

Shu Li

Army Medical University

Zhao Xie ( $\sim$ xiezhao54981@163.com )

Third Military Medical University https://orcid.org/0000-0003-4393-4690

\section{Research article}

Keywords: Femur, Induced membrane technique, Nonunion

Posted Date: August 3rd, 2020

DOl: https://doi.org/10.21203/rs.3.rs-46812/v1

License: (1) This work is licensed under a Creative Commons Attribution 4.0 International License. Read Full License 


\section{Abstract}

Purposes: Large segmental femur bone defect is a serious clinical problem. Limited study have been reported to optimize the fixation during the second stage of induced membrane technique. We would like to report our new findings in two different fixation method with this technique.

Methods: We retrospectively analyzed 56 cases with large segmental femur defects and treated with induced membrane technique from January 2013 to December 2017. Patients were separated into two groups according to different fixation method during the second stage and followed at least 2 years. Bacteria culture, treatment outcome, and complication were collected and analysed.

Results: Patients with an average age of 41.8 years old had an average of 2.6 surgical history. Bone defects were $8.93 \mathrm{~cm}$ in average $(4-16.8 \mathrm{~cm}$ ). Though 20 complications were noticed in this series, $85.7 \%$ $(48 / 56)$ of the cases acquired bone union during their final follow up. However, $57.6 \%(15 / 26)$ of the cases observed obvious bone resorption near the plate in augmented plate group. And this resulted to lower bone union rate was calculated when compared with intramedullary nail group $(P=0.019)$. Insufficient bone grafting material is another main reason resulted nonunion and verified in 5 cases.

Conclusions: Augmented plate will resulted bone resorption and leads to nonunion in induced membrane technique. The plate should be fixated outside the membrane if necessary. Insufficient bone grafting material when grafting is a easily neglectful problem, and proper measures should be taken with cautious.

\section{Background}

Treatment of infected bone defects is a challenge for orthopaedic surgeons[1]. Debridement is the fundamental measure to deal with biofilm which facilitates persistent infection [2]. Repeated operation is very common in clinical scenario, which may result in larger bone defects and difficulty to treat. Ilizarov is a good method but often accompanied with pin infection, nonunion of the docking site and stiffness of adjacent joint[3]. Literatures[4] indicated that induced membrane technique displayed good results and acceptable complications. Very few study cases have been reported to compare different fixation method. Intramedullary nail is preferred during the second stage of this technique, which is superior in smaller bone grafting volume and better bone remodeling. Another advantage is better tolerance and no pin infection for the patients especially in femur, when compared it with external fixation. The issue is that the intramedullary nail is unstable when fixated in large segmental defects. Authors have reported to add another plate and bone grafting in dealing with nonunion after intramedullary nail fixation[5]. We presumed that augmented plate could be useful when instability noticed during the second stage of this technique. Thus, we retrospectively analysed these patients fixated with intramedullary nail or intramedullary nail combined with another augmented plate.

\section{Methods}


We reviewed the clinical database from January 2013 to December 2017, and 83 patients with femur bone defects were treated in our center. According to the inclusion and exclusion criteria, 56 patients were enrolled in this study. The inclusion criteria were patients with large segmental femur bone defects (> 3centimeters) which resulted from infection after debridement or acute bone loss, and treated with induced membrane technique. The exclusion criteria were patients age less than 18 years old or those who did not complete the treatment procedure.

In the first stage operation, hardwares were removed if presented. All the dead bone and infected scarred soft tissue were radically excised(Fig. 1A). Representative tissues were obtained from around the infected site (including the sinus tract) for microbiological cultivation.After radical debridement, the dead space were filled up with antibiotic impregnated PMMA cement spacer ( 0.5 gram gentamycin and 5 gram vancomycin in per 40 gram powder). The cement was applied in its doughy stage in order to form a smooth sheathing. Bone defects were stabilized with unilateral external fixator in 2 cases and antibiotic coated locking compressing plate in other 54 cases(Fig. 1B). Care must be taken when fixated with locking compressing plates, the antibiotic cement needs to coat the implant completely. Antibiotics administration lasted at least 2 weeks till the levels of WBC, ESR and CRP returned to normal limits according to the microbiological cultivation results and susceptibility tests. Postoperatively, partial weight-bearing and physical therapy was instructed.

Bone reconstruction was planned two months later after debridement, when the soft tissue condition is good and normalization of the laboratory values. SPECT was also carried out to exclude possible residual infection. Autologous bone was obtained from posterior iliac rest firstly. And then removing the PMMA spacer and bone grafting. The incision was opened according to the last surgery and microbiological cultures were taken again around the cement. Medullary canal was opened on two sides, the bone defect ends were decorticated and filled up with morselized cancellous autograft obtained from iliac crest or mixed with allograft according to recommended ratio(Fig. 1C). Thirty cases were fixated with intramedullary nail alone. As a precaution of nonunion when instability was noticed, another augmented locking compression plate was added between the defects in 26 patients. Prophylactic antibiotics were administered and continuously for another 2-week after the surgery according to the culture and drug sensitivity tests. Partial weight-bearing was allowed for patients with intramedullary nail and the weight was increased based on the radiographic result of bone healing. Patients were separated into two groups according the fixation method, the intramedullary nail group and augmented locking plate group.

Infection elimination was defined as normalization of laboratory values and resolution of the clinical features of infection (including discharge, redness, swelling, warm and pain). Bone union was defined as grafted bone materials fusion on four cortical side and move without pain(Fig. 1D). Follow-up examinations were carried out with a 4-week interval after the second stage of surgery. Then, a 8 weeks and 16 weeks interval until 2 years after the bone grafting.

\section{Results}


Patients in this study consisted of 10 females and 46 males, with an average age of 41.8 years old (ranged from 18 to 62 years). The causes of the defects are osteomyelitis bone defects after debridement in 51 cases and aseptic nonunion in 5 cases. Patients had an average of 2.6 surgical procedures (range 0-10 procedures) before admitted to our center. Bone defect length were $8.93 \mathrm{~cm}$ in average $(4-16.8 \mathrm{~cm})$ and $85.7 \%(48 / 56)$ of the cases acquired bone union during the final follow up. Amongst them, 51/56 (87.5\%) with infection signs, and intraoperative pathogenic cultures were positive in 36 patients. Twenty complications were noticed in these two group, redebridement in 8 cases, nonunion in 8 cases, second bone grafting in 4 cases.

Augmented locking plate was fixated between the defect when instablility was noticed. Study case presents the external fixator was changed by a antibiotic coated plate during the first stage(Fig. 1A-B) and reconstructed by a intramedullary nail and augmented plate(Fig. 1C). The patient acquired bone union and good functional outcome during his final follow up (Fig. 1D-F). Interestingly, 57.6\%(15/26) of the cases observed obvious bone resorption near the plate in this group (Fig. 2). And this could be found both in bone union patients(Fig. 2A-D) or nonunion patients(Fig. 2E-H). Though most of them acquired bone union, 5 of these cases observed nonunion during their final follow up.There is no differences between ages and bone defect lengths in this two group $(P>0.05)$ (Table 1). Compared with intramedullary nail group, augmented plate group acquired lower bone union rate in this study $(P=0.019)($ Table 2$)$.

Insufficient bone grafting material is another serious problem and noticed in 5 patients. They were encouraged more weight bearing and walking since bone fusion noticed. Dilatory bone union still acquired in 3 patients after 24 months follow up, but another 2 patients failed and separated in each group. Limited by autolougs bone materials, only 11 cases reconstructed with autologus bone in this study. Rest of them were treated with autograft and allograft mixture. Plate fracture also observed in one nonunion case 24 months after the surgery in augmented plate group.

\section{Discussion}

Large segmental femur defect is a big threat in clinical practice. Limited experiences could be noticed after reviewed the database. Ilizarov bone transport considered as the standard protocol[6-7]. However, complication associated with this method is also noticeable[8]. Blum et al[7]treated 50 patients with femoral shaft infected nonunion or bone defects by distraction osteogenesis, and all of them presented pin track infection. Barbarossa et al[3] treated 30 infected femur nonunion cases by llizarov technique and 87 complications encountered in these cases. Options seems to be very inadequate since direct autologous cancellous bone grafting is not suggested when dealing with those bone defects larger than 3- 5 centimeters[9].

Masquelet technique is a relatively easy method. The biological chamber formed by the bone cement obstructs the competitive growth of soft tissues by providing best mechanical environment for bone healing. Tong et al[10] compared 20 patients of lower extremity bone defects treated with Masquelet technique and llizarov bone transport in 19 cases. The author found Masquelet technique showed better 
functional outcomes than llizarov bone transport. In our study, $85.7 \%(48 / 56)$ of the cases acquired bone union during the final follow up. Compared with Azi's study[11], he acquired higher bone union rate (91\%) with this technique. This could be explained by all study cases were large segmental defects. Many papers advised the ratio for allograft and autograft was less than 1:2. Calculating the bone grafting volume is necessary, but accuracy is often affected by unpredictable iliac bone volume and inaccurate bone defect capacity.This miscalculation is difficult to rectify during the final of the surgery, especially in dealing with large segmental bone defects. Delayed union and nonunion often happened in these patients. There were 5 cases observed this problem. Three of them healed and two failed. Benefit from the internal fixation, none of these patients found fixation loosen or unbearable pain during the prolonged bone fusion process.

Antibiotic bone cement have been used in arthroplasty for many years[12]. Then, Conway et al[13] reported 4 cases of osteomyelitis defects treated with antibiotic bone cement coated plate and all of them acquired bone union. The author considered it was a safe method on the basis of radical debridement. Infection could be extinguish ed combined with other auxiliary measures. In this study, locking plates coated with antibiotic cement were applied in 54 cases after debridement. The coated plate effectively reduced the contact surface of the plate and the bacteria contaminated tissue. All of them were satisfied with infection control at their final follow up. Other advantages of this fixation were providing rigidity of the fixation which decrease the possibility of injury the new tiny blood vessel, and increase the comfortability and mobility for the patients as reported[14]. Author also reported[15] infected bone defect cases treated with antibiotic bone cements and acquired bone union.

Apardet et al[16] suggested intramedullary nail as the optimal fixation during the second stage of this technique. Intramedullary nail presents superiority in keeping line of forces, which benefits the bone remodeling and bone union[17]. However, mismatches the nail and the medullary cavities might resulted jiggly move at the fracture site. Such instability often resulted nonunion in large segmental defect cases[18]. In this study, augmented plate was initially added in intramedullary nail group after nonunion. Two of them were fixated with another plate without removing the nail and acquired bone union. Inspired by this, cases with possibility of nonunion were fixated with intramedullary nail and augmented plate. But $57.6 \%(15 / 26)$ of the augmented plate cases noticed bone resorption near the plate. Though most cases acquired bone union, 5 of them observed nonunion during their final follow up. And higher bone union rate was calculated in intramedullary nail group. This might be due to the augmented plate separated the induced membrane and the grafting material. The solution for this problem is the membrane should be sutured firstly(Fig. 3A-B). We consider for patients with too short residual bone segments in distal femur(Fig. 1C) or have a large difference in diameter after reaming should be reconstructed with another augmented plate to eliminate the jiggle of the fracture site. But the plate should be fixated outside the induced membrane.

\section{Conclusions}


Intramedullary nail is central fixation and superior in reducing bone materials, and complications as pin infection and fixation loosen when delayed bone healing happened. But this method is often unstable when dealing with distal femur or large segmental defects.Augmented plate can provide more holding force to facilitate bone healing. However, the plate separated the membrane and the grafting materials which contradicted with the essence of induced membrane technique. And this contradiction might resulted more nonunion.Thus, the plate should be fixated outside the membrane when the membrane be sutured if necessary.Besides, insufficient bone grafting material is also another reason lead to nonunion and easily neglectful. Proper measures should be taken with cautious.

List of

\section{Declarations}

\section{Ethics approval and consent to participate}

Ethics approval was obtained from the Ethics Committee of the First Affiliated

\section{Consent for publication}

All presentations provided consent for publication.

\section{Competing interests}

We declare that there are no conflicts of interest with any institution.

\section{Funding}

This work was supported by by the Major Military Logistics Research Projects (grant no. AWS17J004-0205). There is not any commercial sources to help design or collection or writing for this manuscript.

\section{Authors' contributions}

SY contributed to writing the paper.BY and SW contributed to collecting the data and analysing the data. CJ associated with ZX performed the surgeries. LS contributed to revising the manuscript. ZX contributed to overall planning and performed the surgeries.

\section{Acknowledgements}


None

\section{Abbreviations}

Cm,centimeters; PMMA,Polymethyl methacrylate;WBC, white blood cell, ESR, erythrocyte sedimentation rate; CRP,C-reactive protein;SPECT, Single-Photon Emission Computed Tomography

\section{References}

1. Eralp LM Kocaoglu, and Rashid H(2007) Reconstruction of segmental bone defects due to chronic osteomyelitis with use of an external fixator and an intramedullary nail. Surgical technique. J Bone Joint Surg Am, 89 Suppl 2 Pt.2: p. 183-95.doi: 10.2106/JBJS.G.00306.

2. Simpson AH. Deakin M, and Latham JM. (2001) Chronic osteomyelitis. The effect of the extent of surgical resection on infection-free survival. Journal of Bone Joint Surgery-British Volume, 83(3) $p$. 403-7.doi: 10.1302/0301-620x.83b3.10727.

3. Barbarossa V. Matković BR, Vucićet N. Bielen M,Gluhinić M(2001) Treatment of osteomyelitis and infected non-union of the femur by a modified Ilizarov technique: follow-up study. Croat Med J, 42(6): p. 634-641..

4. Mauffrey C. Hake ME, Chadayammuri V. Masquelet AC(2016) Reconstruction of Long Bone Infections Using the Induced Membrane Technique: Tips and Tricks. J Orthop Trauma, 30(6) $p$. e188-93. doi. 10.1097/BOT.0000000000000500.

5. Yang KH. Won Y, Kim SB. Oh BH,Park YC. Jeong SJ(2016) Plate augmentation and autologous bone grafting after intramedullary nailing for challenging femoral bone defects: a technical note. Arch Orthop Trauma Surg 136(10): 1381-5.doi: 10.1007/s00402-016-2522-9.

6. Krishnan A. Pamecha C and Patwa JJ(2006) Modified llizarov technique for infected nonunion of the femur: the principle of distraction-compression osteogenesis. J Orthop Surg, 14(3) p. 265-72.dor. $10.1177 / 230949900601400307$.

7. Blum AL. BongioVanni JC, Morgan SJ,Flier MA, Reis FBD. (2010) Complications associated with distraction osteogenesis for infected nonunion of the femoral shaft in the presence of a bone defect: a retrospective series. Journal of Bone Joint Surgery-British Volume, 92(4) p. 565-70.dor. 10.1302/0301-620X.92B4.23475.

8. Gelalis ID,Politis AP,Arnaoutoglou CM,Korompilias AV,Pakos EE,Vekris MD,Karageorgos A,Xenakis TA. (2012) Diagnostic and treatment modalities in nonunions of the femoral shaft: a review. InjuryInternational Journal of the Care of the Injured, 43(7) p. 980-8. doi. 10.1016/j.injury.2011.06.030.

9. Mauffrey C, Barlow BT and Smith,W(2015) Management of segmental bone defects. J Am Acad Orthop Surg, 23(3) p. 143-53.doi. 10.5435/JAAOS-D-14-00018.

10. Tong K,Zhong ZY,Peng YL,Lin CX,Cao SL,Yang YP,Wang G. (2017) Masquelet technique versus llizarov bone transport for reconstruction of lower extremity bone defects following posttraumatic 
osteomyelitis. Injury-International Journal of the Care of the Injured, 48(7) p. 1616-22.dor. 10.1016/j.injury.2017.03.042.

11. Azi ML,Teixeira AAA, Cotias RB,Joeris A, Jr MK (2016) Membrane Induced Osteogenesis in the Management of Posttraumatic Bone Defects. J Orthop Trauma, 30(10) p. 545-50.doi. 10.1097/BOT.0000000000000614.

12. Trippel SB(1986) Antibiotic-impregnated cement in total joint arthroplasty. Journal of Bone and Joint Surgery-American Volume, 68(8): p. 1297-1302.

13. Conway JD, Hlad LM, Bark SE. (2015) Antibiotic cement-coated plates for management of infected fractures. American journal of orthopedics (Belle Mead, $N$ J), 44(2): p. E49-53.

14. Yu X. Wu H, Li J. Xie Z(2017) Antibiotic cement-coated locking plate as a temporary internal fixator for femoral osteomyelitis defects. Int Orthop,41(9):1851-7.doi: 10.1007/s00264-016-3258-4.

15. Scholz AO,Gehrmann S,Glombitza M,Kaufmann RA,Bostelmann R,Flohe S, Windolf J(2015) Reconstruction of septic diaphyseal bone defects with the induced membrane technique. InjuryInternational Journal of the Care of the Injured 46 Suppl 4: p. 121-4. doi: 10.1016/S00201383(15)30030-9.

16. Apard T,Bigorre N,Cronier P,Duteille F,Bizot P,Massin P(2010) Two-stage reconstruction of posttraumatic segmental tibia bone loss with nailing. Orthopaedics traumatology surgery research: OTSR, 96(5) p. 549-53.doi: 10.1016/j.otsr.2010.02.010.

17. Singh AK, Narsaria N, Gupta RK. (2017) A biomechanical study comparing proximal femur nail and proximal femur locking compression plate in fixation of reverse oblique proximal femur fractures. Injury-International Journal of the Care of the Injured, 48(10): p. 2050-2053. doi: 10.1016/j.injury.2017.05.029.

18. Vaishya R. Agarwal AK, Gupta N. Vijay V(2016) Plate augmentation with retention of intramedullary nail is effective for resistant femoral shaft non-union. $J$ Orthop, 13(4) p. 242-5. doi: 10.1016/j.jor.2016.06.003. eCollection 2016 Dec.

\section{Tables}

Table.1 Statistics of two groups between age and bone defect length.

$$
\text { Age Length }
$$

\begin{tabular}{lcc}
\hline IN group $(\mathrm{n}=30)$ & $39.83 \pm 14.79$ & $8.23 \pm 3.80$ \\
AP group $\square \mathrm{n}=26 \square$ & $44.08 \pm 11.42$ & $9.73 \pm 2.73$ \\
T value & -1.188 & -1.710 \\
P value & 0.240 & 0.093 \\
\hline
\end{tabular}

*intramedullary nail (IN), augmented plate (AP)

Table.2 Statistics of two groups between nonunion rate. 


\begin{tabular}{lccc}
\hline & Bone union & Nonunion & P value \\
\hline IN group $(\mathrm{n}=30)$ & 29 & 1 & \\
AP group $[\mathrm{n}=26[$ & 19 & 7 & \\
& & & 0.019
\end{tabular}

*intramedullary nail (IN), augmented plate (AP)

\section{Figures}
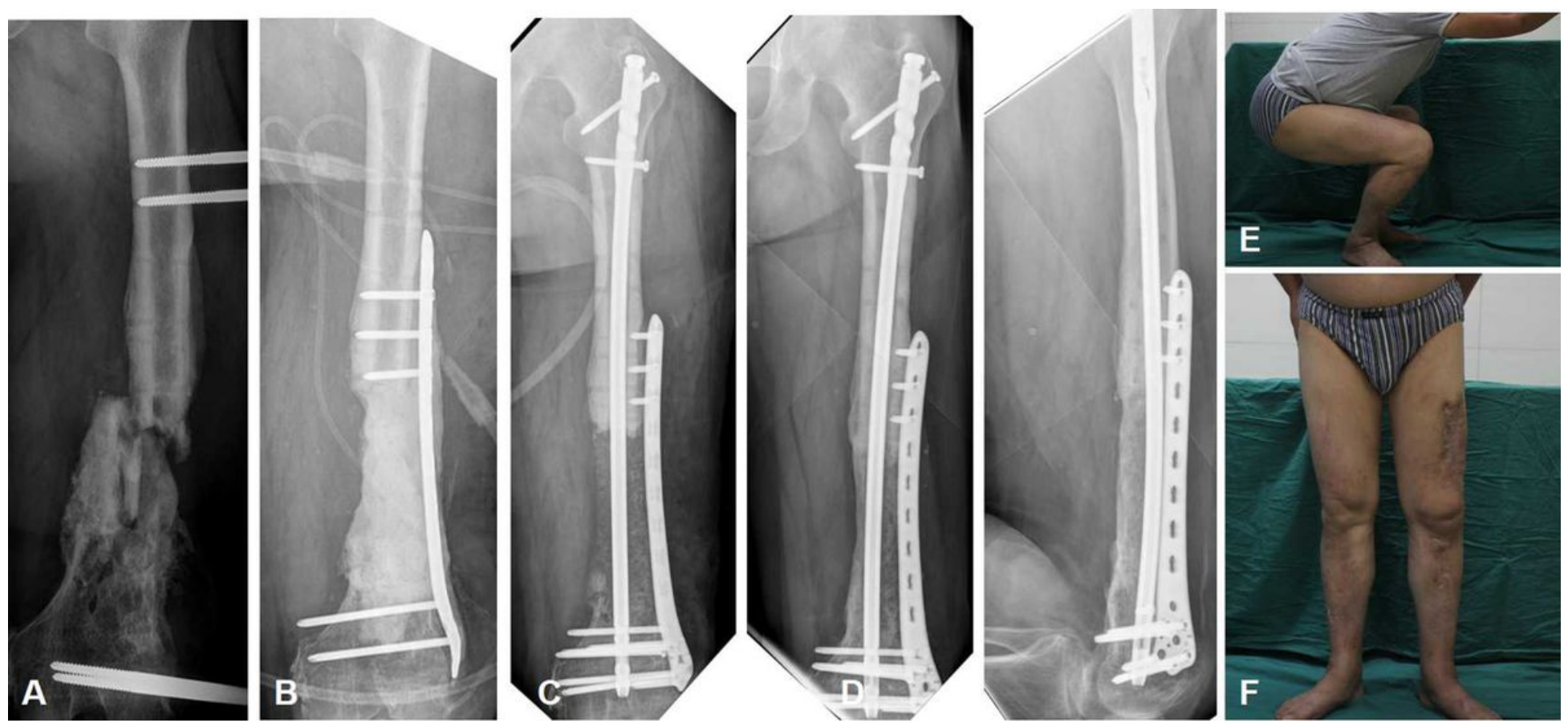

Figure 1

Study case presents a 53 years old male suffered from chronic osteomyelitis treated with induced membrane technique. Fig.1A Anteroposterior X-ray before the surgery; Fig.1B X-ray after the debridement surgery, external fixator was replaced by antbibiotic bone cement coated plate; Fig.1C Bone cement was removed and grafted with autologous bone; Fig.1D Anteroposterior and lateral view of the X-ray after 24 months follow up, bone union were acquired on both medial and lateral sides of the femur. Fig.1E-F Functional outcome of the patient after $\mathbf{2 4}$ months of the surgery. 

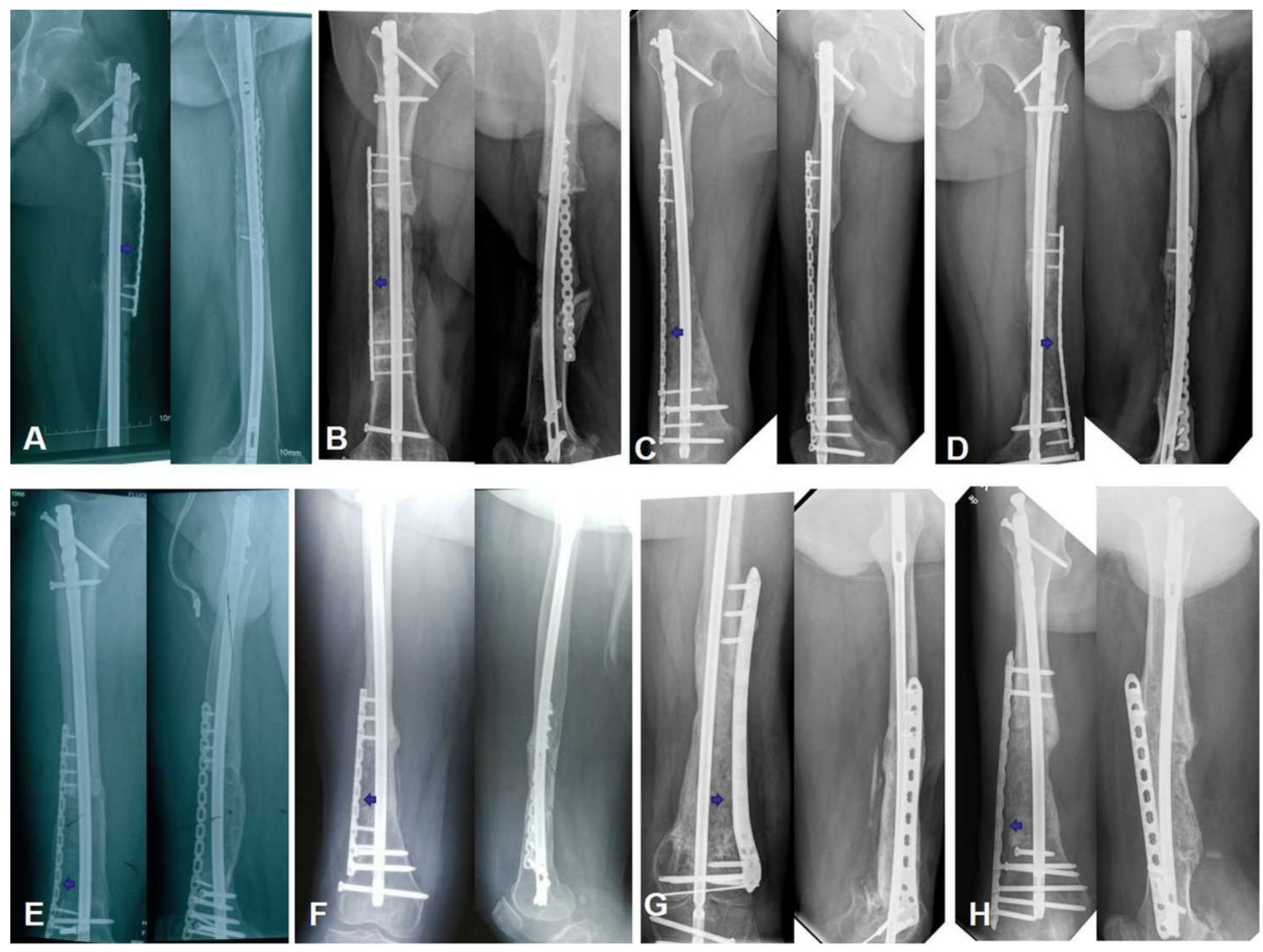

Figure 2

Bone resorption near the augmented plate in 4 nonunion and 4 union patients. Fig.2A-D Anteroposterior and lateral X-ray of 4 nonunion patients during their final follow up. Obvious bone resorption at the plate side can be noticed. Fig.2E-H Anteroposterior and lateral X-ray of 4 bone union patients in augmented plate group. Same bone resorption at the plate side can be found. The blue arrow demonstrated where bone resorption observed. 

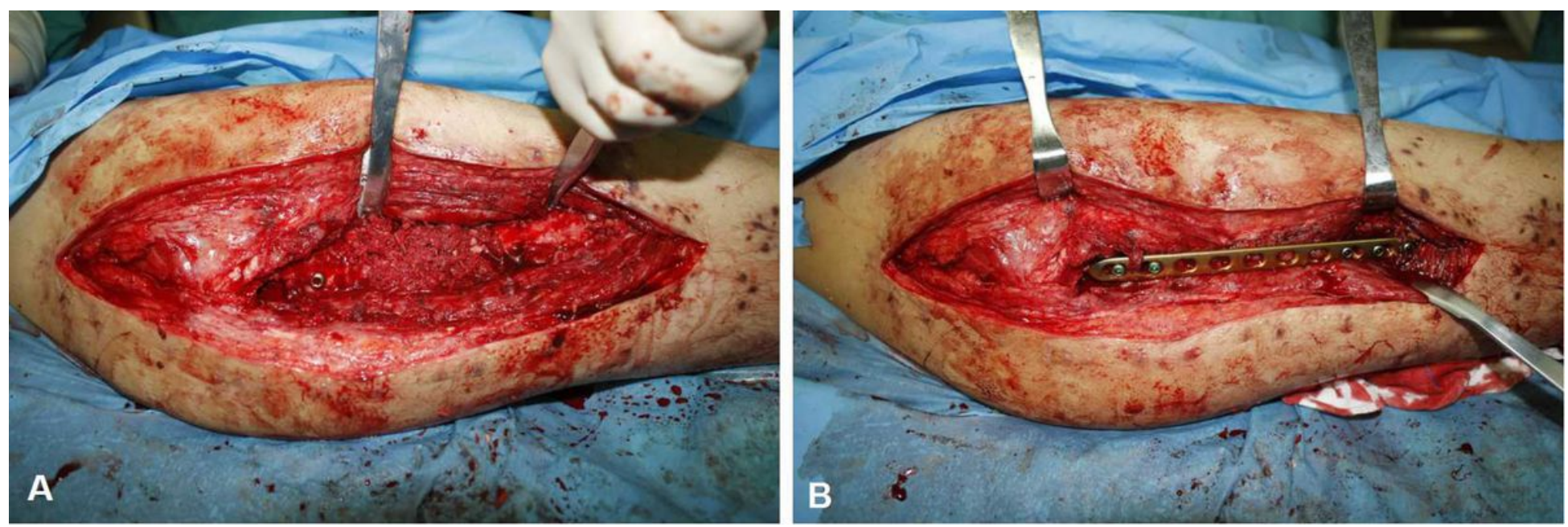

Figure 3

Advisable solution for bone resorption near the plate. A Bone grafting was carried out before fixated with augmented plate; $B$ The plate was added after induced membrane and the muscle were sutured 